\title{
Biogenic gas nanostructures as ultrasonic molecular reporters
}

\author{
Mikhail G. Shapiro ${ }^{1,2,3 \star}$, Patrick W. Goodwill2 ${ }^{2}$ Arkosnato Neogy ${ }^{4}$, Melissa Yin ${ }^{5}$, F. Stuart Foster ${ }^{5,6}$, \\ David V. Schaffer ${ }^{2,7}$ and Steven M. Conolly ${ }^{2,4}$
}

\begin{abstract}
Ultrasound is among the most widely used non-invasive imaging modalities in biomedicine ${ }^{1}$, but plays a surprisingly small role in molecular imaging due to a lack of suitable molecular reporters on the nanoscale. Here, we introduce a new class of reporters for ultrasound based on genetically encoded gas nanostructures from microorganisms, including bacteria and archaea. Gas vesicles are gas-filled protein-shelled compartments with typical widths of $45-250 \mathrm{~nm}$ and lengths of $100-600 \mathrm{~nm}$ that exclude water and are permeable to gas ${ }^{2,3}$. We show that gas vesicles produce stable ultrasound contrast that is readily detected in vitro and in vivo, that their genetically encoded physical properties enable multiple modes of imaging, and that contrast enhancement through aggregation permits their use as molecular biosensors.
\end{abstract}

Contrast-enhanced ultrasound has been an active area of research and development for over 40 years $^{4}$, with conventional ultrasound contrast agents formulated as lipid- or protein-stabilized gas microbubbles. The partial pressure gradient between a microbubble's gas interior and the surrounding media, which is inversely proportional to its radius, limits most microbubbles to sizes larger than $1 \mu \mathrm{m}$ and leads to gas escape, bubble fragmentation and collapse after in vivo administration ${ }^{5,6}$. Although microbubbles have been very successful in clinical imaging of the blood pool and related physiology ${ }^{7}$ and have recently been proposed as potential cell-internalized labels ${ }^{8}$, several factors limit their range of applications in molecular imaging. For example, microbubbles of micrometre size are typically limited to imaging targets within the vasculature. In addition, microbubbles typically have a short halflife compared to the in vivo dynamics of immune, stem and other cells $^{5,7,9}$. Furthermore, none of the solid ${ }^{10}$, liquid ${ }^{11}$, hollow ${ }^{12}$ or phase-change ${ }^{13}$ contrast agents proposed as microbubble alternatives have so far become widely adopted due to limitations in echogenicity, stability or ease of synthesis. As a result, important studies in oncology, immunology, regenerative medicine and other biomedical areas remain dominated by nanoparticle reporters designed for optical, magnetic resonance and nuclear imaging ${ }^{14}$.

We hypothesized that nanoscale reporters with novel properties for molecular ultrasonography could be derived from natural biological structures. In particular, we considered gas vesicles, genetically encoded gas nanostructures formed by certain bacteria and archaea as a means to control buoyancy for optimal access to light and nutrients ${ }^{2,3}$. Gas vesicles have cylindrical or biconical shapes, with maximal diameters of $45-250 \mathrm{~nm}$ and typical lengths of $100-600 \mathrm{~nm}$, varying between genetic hosts ${ }^{2,3}$. These nanostructures interact with gases through a mechanism that is fundamentally different from that in microbubbles. Whereas microbubbles trap pre-loaded gas, gas vesicles exclude water but permit gas from the surrounding media to freely diffuse in and out of their 1-10 al interior through a $2 \mathrm{~nm}$ protein $\operatorname{shell}^{2}$ (Fig. 1a). As a result, no pressure gradient exists between the inside and outside of gas vesicles, permitting them to be inherently stable despite their nanometre size. Although they were discovered over 100 years ago $^{15}$ and have been well-characterized biophysically ${ }^{2}$, gas vesicles have yet to be substantially exploited as a nanotechnology ${ }^{3}$. We hypothesized that such gas vesicles could serve as nanoscale molecular reporters for ultrasound imaging and, furthermore, that their genetically encoded structural properties, such as collapse at specific hydrostatic pressures, could enable new imaging modes beyond those available with microbubbles.

To test this hypothesis, gas vesicles from Anabaena flos-aquae (Ana) and Halobacterium NRC-1 (Halo) (Fig. 1b,c), representing two genetically distant sources ${ }^{3}$, were purified through tonic cell lysis and centrifugally assisted flotation, and imaged in gel phantoms using a scanning single-element ultrasound imaging system operating at 4.8, 8.6 and $17 \mathrm{MHz}$ (for sample preparation and imaging details, see Methods). Gas vesicles from both species produced robust contrast relative to buffer controls at optical densities (OD) ranging from 0.25 to 2.0 (Fig. $1 \mathrm{~d}-\mathrm{g}$ ), corresponding to nanostructure concentrations of $150 \mathrm{pM}$ to $1.2 \mathrm{nM}($ Ana) or $5 \mathrm{pM}$ to $40 \mathrm{pM}$ (Halo) and gas volume fractions of $\sim 0.01-0.1 \%$. Gas vesicle echogenicity in this configuration was detectable for over one week (Supplementary Fig. 1). Contrast was strongest at the highest frequency, with OD 2.0 Ana gas vesicles producing $27.0 \pm 4.1$ greater scattering than buffer controls (Fig. 1f). The balance of scattering and attenuation differed between Ana and Halo gas vesicles. Whereas Ana gas vesicles produced backscatter fairly uniformly along the axial dimension (from top to bottom in Fig. 1d), Halo gas vesicles at higher concentrations produced acoustic attenuation, manifested as a reduced signal beneath the vesicles in the gel phantom (Fig. 1e, Supplementary Fig. 2).

Gas vesicles can be collapsed by rapidly increasing the hydrostatic pressure past a species-specific critical point, ranging from $40 \mathrm{kPa}$ to over $700 \mathrm{kPa}$ (refs 2,16) (Fig. 1b,c). Gas vesicles that were hydrostatically collapsed before loading into the phantom failed to show ultrasound contrast, confirming the echogenic role of their gas compartments (Fig. 1d,e).

Nonlinear imaging modes relying on harmonic signals have been used to improve the contrast specificity of microbubble-enhanced

\footnotetext{
${ }^{1}$ Miller Research Institute, University of California at Berkeley, 2536 Channing Way, Berkeley, California 94720, USA, ${ }^{2}$ Department of Bioengineering, 306 Stanley Hall MC \#1762, University of California at Berkeley, Berkeley, California 94720, USA, ${ }^{3}$ Department of Molecular and Cell Biology, 142 LSA \#3200, University of California at Berkeley, Berkeley, California 94720, USA, ${ }^{4}$ Department of Electrical Engineering and Computer Science, University of California at Berkeley, Berkeley, California 94720, USA, ${ }^{5}$ Sunnybrook Research Institute, University of Toronto, 2075 Bayview Avenue, Toronto, Ontario M4N 3M5, Canada, ${ }^{6}$ Department of Medical Biophysics, University of Toronto, 610 University Avenue, Toronto, Ontario M4N 3M5, Canada, ${ }^{7}$ Department of Chemical and Biomolecular Engineering, University of California at Berkeley, Berkeley, California 94720, USA. Present address: Division of Chemistry and Chemical Engineering, California Institute of Technology, 1200 East California Boulevard, Pasadena, California 91125, USA. *e-mail: mikhail@caltech.edu
} 

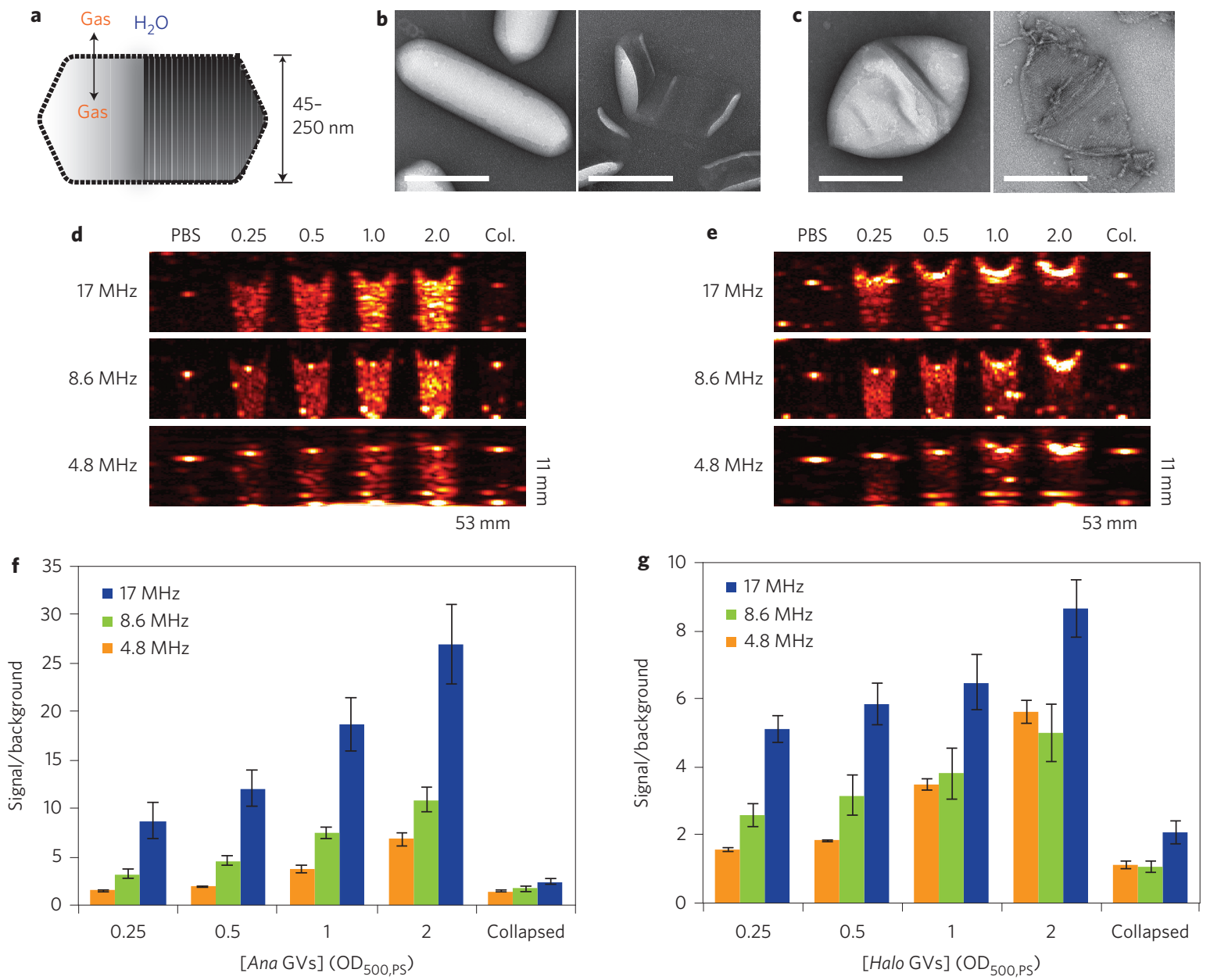

Figure 1 | Gas vesicles produce ultrasound contrast. a, Diagram of a gas vesicle: a hollow gas nanocompartment (solid shading) surrounded by a gaspermeable protein shell (ribbed shading). b, TEM images of intact (left) and hydrostatically collapsed (right) Ana gas vesicles. c, TEM images of intact (left) and collapsed (right) Halo gas vesicles. All scale bars, $200 \mathrm{~nm}$. d, Ultrasound images of a gel phantom containing PBS buffer, Ana gas vesicles at optical densities ranging from OD 0.25 to 2 (concentrations of $150 \mathrm{pM}$ to $1.2 \mathrm{nM}$ ) or collapsed Ana gas vesicles (OD 2.0). Images were acquired at multiple frequencies, as indicated. e, Ultrasound images of a gel phantom containing PBS buffer, Halo gas vesicles at optical densities ranging from OD 0.25 to 2 (concentrations of 5 to $40 \mathrm{pM}$ ) or collapsed Halo gas vesicles (OD 2.0). Conversion between OD, molar concentration and gas volume fraction is described in the Methods. The size of each field of view is indicated in the lower right corner of the images. $\mathbf{f}$, Total backscattered signal relative to PBS at each frequency and Ana gas vesicle (GV) concentration ( $N=4$ per sample). g, Total backscattered signal relative to PBS at each frequency and Halo gas vesicle concentration ( $N=4$ per sample). Detailed image acquisition and analysis parameters are provided in Supplementary Table 1, and colour maps for ultrasound images in Supplementary Fig. 9. All error bars represent \pm standard error of the mean (s.e.m.).

ultrasound ${ }^{17}$. In particular, harmonic backscatter is thought to arise from oscillations in microbubble radius ${ }^{18}$ in response to incident pressure waves. We tested whether harmonics could also be detected from gas vesicles. Transmitting at $6 \mathrm{MHz}$, we observed substantial second- and third-harmonic signals in Halo gas vesicles at $12 \mathrm{MHz}$ and $18 \mathrm{MHz}$ compared to polystyrene microspheres, a linear scattering reference material (Fig. 2a). Ana gas vesicles did not produce harmonic signals at $6 \mathrm{MHz}$ (Supplementary Fig. 3). Images formed by processing Halo gas vesicle signals through bandpass filters centred at the second and third harmonics showed 3.7-fold and 4.6-fold greater contrast enhancement in vesicle-containing wells relative to the linear scatterer than did images processed at the transmitted frequency (Fig. 2b,c). To further explore gas vesicle-specific contrast enhancement, we took advantage of pressure-induced vesicle collapse by testing its application in situ at ultrasound frequencies. Halo and Ana gas vesicles were imaged before and after a disruptive scan at 8.6 $\mathrm{MHz}$ in which the transducer output was set to produce a peak pressure of $650 \mathrm{kPa}$-above the critical collapse level of both species of vesicles $(70-150 \mathrm{kPa}$ for Halo and $440-605 \mathrm{kPa}$ for $A n a)^{16}$ (Fig. 2d). The gas vesicle signal was mostly eliminated, enabling the generation of subtraction images in which the signal from Halo and Ana gas vesicle wells relative to polystyrene was boosted by factors of 10 and 22, respectively (Fig. 2d,e). Such subtraction images can be used to increase the specificity of contrast information relative to background scattering ${ }^{19}$.

The fact that gas vesicles from different species have distinct critical collapse pressures ${ }^{16}$ raised the further prospect of distinguishing multiple populations of vesicles in the same sample through 'serial collapse' imaging. In this paradigm, contrast originating from gas vesicles with a low collapse pressure is identified by its disappearance upon the application of pressure pulses with amplitudes above their critical point but below that of other vesicles. The remaining contrast is attributable to gas vesicle species with higher collapse pressures. To test this paradigm with our two species of vesicles, we prepared imaging phantoms with wells containing Halo gas vesicles, Ana gas vesicles, or a combination of both (Fig. 2f). In an initial image, both populations of vesicle produce contrast (Fig. $2 \mathrm{f}$, top), but after low-pressure collapse at $300 \mathrm{kPa}$, contrast in the Halo gas vesicle well is eliminated and contrast in the mixed well is reduced (Fig. $2 \mathrm{f}$, middle). After high-pressure collapse at $650 \mathrm{kPa}$, contrast in the 

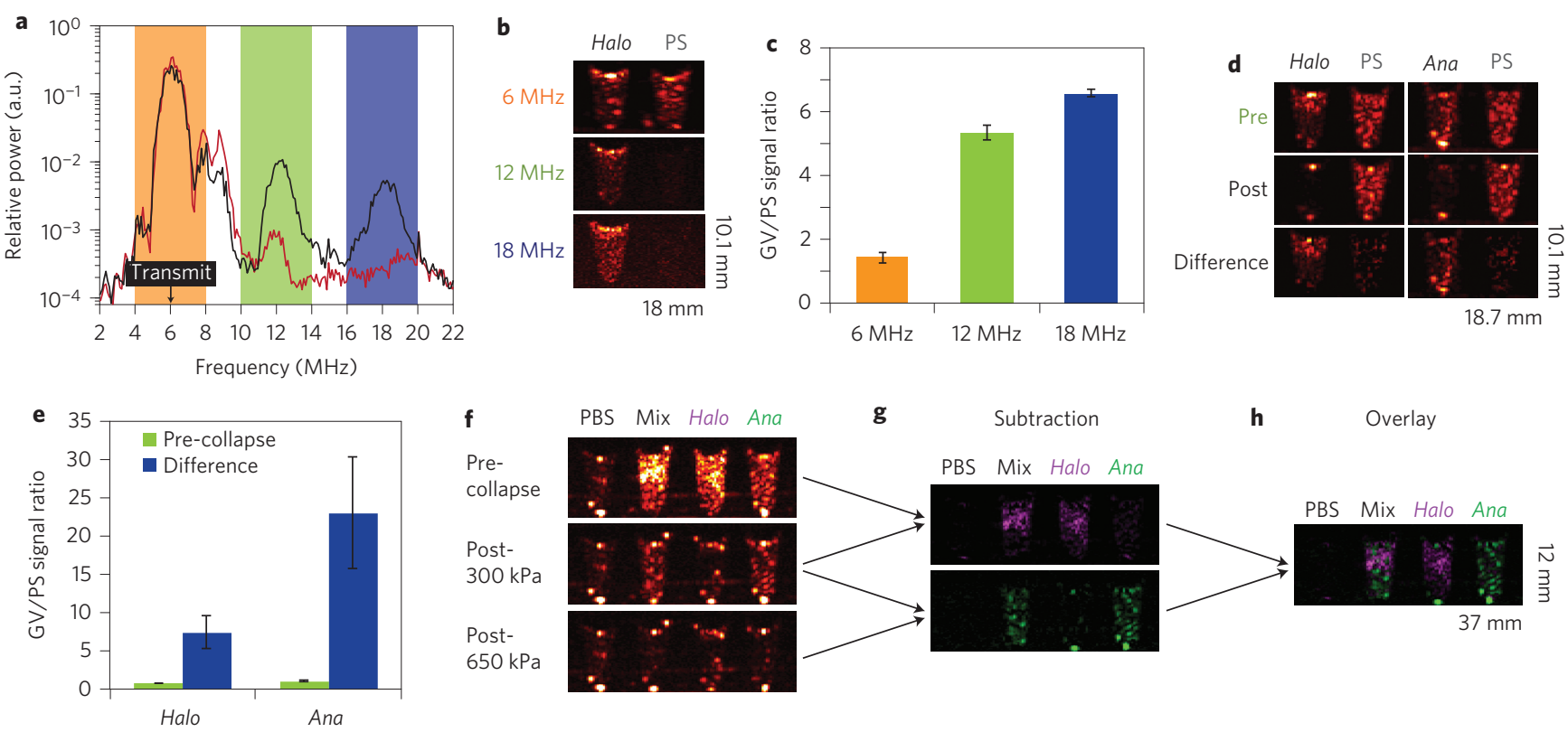

Subtraction

h

Overlay

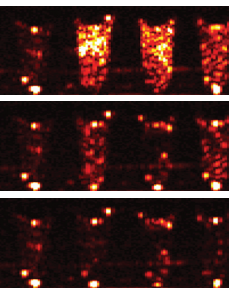

PBS Mix Halo Ana

Figure 2 | Nonlinear imaging and genetic diversity enable enhanced contrast specificity and selective disruption imaging. a, Power spectrum of signal backscattered from Halo gas vesicles (black) and $4.78 \mu \mathrm{m}$ polystyrene (PS) microspheres (red) in response to $6 \mathrm{MHz}$ transmitted pulses (peak amplitude $98 \mathrm{kPa}$, labelled 'Transmit' in the figure). Each point on the spectrum represents an average of 48 points from three samples (16 points per sample). The orange, green and blue shaded areas correspond to frequency bands used to generate the images in $\mathbf{b}$. $\mathbf{b}$, Ultrasound images of Halo gas vesicles and PS microspheres acquired with $6 \mathrm{MHz}$ transmission and bandpass-filtered around 6, 12 and $18 \mathrm{MHz}$. c, Ratio of total backscattered signal from Halo gas vesicles and PS microspheres after filtering at the indicated frequencies $(N=4)$. d, Ultrasound images of Halo gas vesicles, Ana gas vesicles and PS microspheres at $8.6 \mathrm{MHz}$ acquired before (Pre) and after (Post) destructive collapse with $650 \mathrm{kPa}$ insonation, and the difference (Difference) between these images. e, Ratio of total backscattered signal from gas vesicles and PS microspheres in pre-collapse and difference images $(N=4)$. The concentrations used in a-e were OD 0.5 Halo gas vesicles, OD 2.0 Ana gas vesicles and 0.83\% (wt/vol) polystyrene. f, Ultrasound images of a phantom containing wells with PBS, a mixture of Ana and Halo gas vesicles, or each type of gas vesicle on its own (all gas vesicles at OD 1.0 in PBS), acquired at 8.6 MHz. Top: before collapse. Middle: after collapse at 300 kPa. Bottom: after collapse at 650 kPA. g, Top: Difference between the top and middle images in f. Bottom: Difference between the middle and bottom images in $\mathbf{f}$. $\mathbf{h}$, Overlay of the two images in $\mathbf{g}$. Detailed image acquisition and analysis parameters are provided in Supplementary Table 1, and colour maps for ultrasound images in Supplementary Fig. 9. The size of each field of view is indicated in the lower right corner of the images. All error bars represent \pm s.e.m.

Ana gas vesicle well and the mixed well is also eliminated, leaving only background (Fig. 2f, bottom). Halo gas vesicle contrast can now be inferred by subtracting the second image from the first image (Fig. 2g, top) and Ana gas vesicle contrast can be calculated by subtracting the third image from the second image (Fig. $2 \mathrm{~g}$, bottom). An overlay correctly shows the mixed well as containing both types of vesicle (Fig. $2 \mathrm{~h}$ ).

Because gas vesicles are smaller than the wavelengths typically used in ultrasound, we further hypothesized that assemblies of gas vesicle nanoparticles would produce enhanced scattering compared to unaggregated solutions, as previously shown with perfluorocarbon droplets ${ }^{11}$, potentially enabling gas vesicles to serve as biomolecular sensors analogous to aggregation-dependent nanoparticle reporters for magnetic resonance imaging $(\mathrm{MRI})^{20}$. To test this possibility, we functionalized the surface of Ana gas vesicles with biotin and exposed them to various quantities of free streptavidin. In the absence of streptavidin, or at streptavidin concentrations sufficient to saturate surface biotins, the vesicles are expected to remain unaggregated. However, at intermediate concentrations, streptavidin is expected to mediate gas vesicle clustering (Fig. 3a). The formation of aggregates $(\sim 1 \mu \mathrm{m})$ in response to streptavidin was confirmed by transmission electron microscopy (TEM; Fig. 3d) and at higher magnification it was also possible to discern individual streptavidin molecules on the surface of the gas vesicles at the expected relative densities (arrows in Fig. 3d). When nanoparticle assemblies were imaged in ultrasound, the intermediate streptavidin concentration was detected by a twofold increase in contrast (Fig. 3b,c). Ana gas vesicles were chosen for functionalization because their greater resistance to collapse (compared to Halo vesicles) allows easier handling during repeated purifications.

Next, to test the feasibility of using gas vesicles expressed inside cells as intracellular reporters or genetic labels, we imaged intact Ana cells and compared the resulting contrast to vesicles that were released from the same quantity of cells through hypertonic lysis (Fig. 3e-g). The intact cells exhibited a 12-fold stronger signal, indicating that intracellular gas vesicles have the potential to serve as genetically encoded reporters or dynamic indicators of cellular integrity. The quantity of gas vesicles recovered after lysis $(815 \pm 124 \mu \mathrm{l}$ gas volume per gram of dry cell pellet, $N=4)$ was greater than previously measured intracellular gas vesicle contents $\left(\sim 350-500 \mu \mathrm{g}^{-1}\right)^{21,22}$, suggesting that the observed difference in contrast between intact and lysed cells is unlikely to arise from vesicle destruction during lysis.

Finally, to demonstrate that gas vesicles are capable of producing ultrasound contrast in vivo, we performed subcutaneous and intravenous injections of Halo gas vesicles into live anaesthetized mice, choosing Halo vesicles for their robust nonlinear signals (Fig. 2a). We first injected the gas vesicles or a buffer control subcutaneously into the lower abdomen of anaesthetized CD-1 mice and acquired second-harmonic images (transmitting at $6 \mathrm{MHz}$ ) using our single-element apparatus. We observed robust enhancement on the gas vesicle side (Fig. 4a,b,d), but not on the control side. To confirm that the vesicles were the source of the observed contrast, we applied ultrasonic pulses at a supracollapse pressure $(650 \mathrm{kPa})$, 

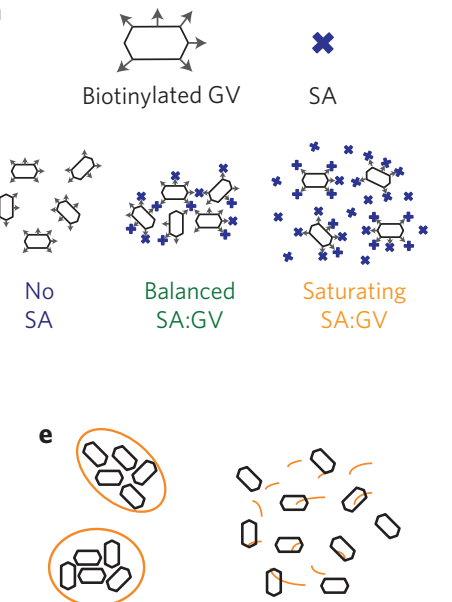

Intact cells

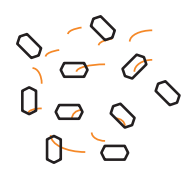

Lysed cells

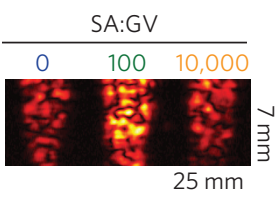

f

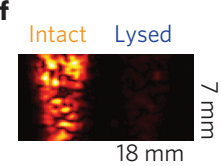

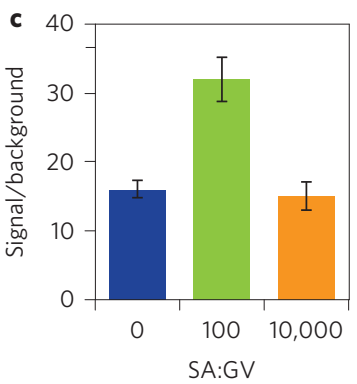

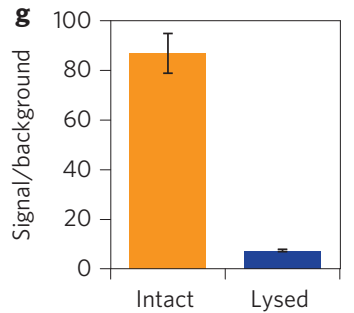

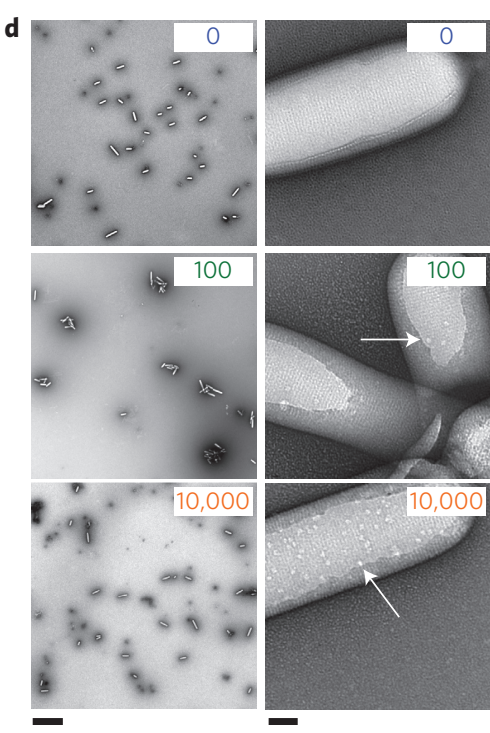

Figure 3 | Gas vesicles act as biomolecular sensors and report cellular integrity. a, Illustration of predicted aggregation interactions between surfacebiotinylated gas vesicles (hexagons with grey arrows) and streptavidin (SA) at different SA:GV ratios. b, An image, acquired at 17 MHz, of OD 1.0 biotinylated Ana gas vesicles mixed with the indicated ratio of streptavidin. c, Integrated signal intensity relative to phantom background corresponding to the SA:GV conditions in $\mathbf{b}(N=4$ per condition). d, TEM images of Ana gas vesicles incubated with streptavidin at the indicated molar ratios on the top right-hand corner of each panel. At the higher magnification (right), arrows indicate apparent streptavidin molecules on the vesicle surface. Scale bars, $2 \mu m$ (left) and $40 \mathrm{~nm}$ (right). e, Illustration of gas vesicles (black hexagons) confined inside intact cells (orange) or released following lysis. $\mathbf{f}$, Ultrasound image (17 $\mathrm{MHz}$ pulses) of Ana cells treated with water (intact) or with $25 \%$ sucrose (lysed). $\mathbf{g}$, Integrated signal intensity relative to phantom background for intact and lysed cells ( $N=4$ per condition). Detailed image acquisition and analysis parameters are provided in Supplementary Table 1, and colour bars for ultrasound images in Supplementary Fig. 9. The size of each field of view is indicated in the lower right corner of the images. All error bars represent \pm s.e.m.

resulting in contrast disappearance (Fig. 4c,d). Regions of interest containing gas vesicles exhibited $60 \pm 14 \%$ stronger backscattered signals than buffer-injected controls $(P=0.008)$; this difference disappeared after collapse $(P=0.23)$. Images from five animals are shown in Supplementary Fig. 4. In addition, we used a commercial high-frequency small-animal ultrasound scanner operating at $18 \mathrm{MHz}$ to image the dynamics of Halo gas vesicles $(50 \mu \mathrm{l}$, OD 6.0) injected into the tail vein of severe-combined immunodeficient (SCID) nude mice. Five seconds after the start of the injection (at a rate of $0.6 \mathrm{ml} \mathrm{min}{ }^{-1}$ ), robust scattering was observed from the inferior vena cava (IVC) in nonlinear contrast images (Fig. 4e,h,i and Supplementary Movie 1). Then, as expected for unfunctionalized nanostructures, gas vesicle contrast accumulated in the liver, reaching a steady state after $\sim 50 \mathrm{~s}$ (Fig. $4 \mathrm{f}, \mathrm{h}, \mathrm{j}$ and Supplementary Movie 1). Application of a high-power burst from the transducer completely eliminated the accumulated contrast (Fig. 4 g,h,i,j and Supplementary Movie 1). Under these conditions, gas vesicles were detectable in vivo when injected at concentrations as low as OD 1.5 in the IVC (Fig. 4k), and OD 3.0 in the liver (Fig. 4l); contrast increased with dosing above these levels.

No acute toxicity was observed in the intravenously injected mice. The animals' heart rates and breathing remained normal during and after imaging (Supplementary Fig. 5), and no significant decrease in five veterinary health measures (activity, weight, food intake, posture and hydration) was observed immediately or 24 and $48 \mathrm{~h}$ after vesicle administration (Supplementary Table 2). Gas vesicles taken up by the liver appeared to be degraded within $60 \mathrm{~min}$ of uptake (Supplementary Fig. 6), consistent with other protein nanostructures cleared by this organ, such as bacteriophage ${ }^{23}$. However, when vesicles were injected subcutaneously, occupying a tissue that lacks rapid nanoparticle clearance and protein degradation, they produced stable contrast for at least $2 \mathrm{~h}$ (Supplementary Fig. 7).

Our findings establish gas vesicles as a promising new class of molecular reporters for ultrasonography. Their ability to produce stable contrast at subnanomolar reporter concentrations may enable future applications of ultrasound in imaging studies that, to date, have been dominated by nanoparticle reporters for other modalities such as MRI and fluorescence ${ }^{14}$. These concentrations represent gas contents $(<1 \mu \mathrm{lgas} / \mathrm{ml}$ solution) similar to those of typical formulations of microbubbles, which contain $\sim 1,000$ times more gas per particle $^{19}$. Furthermore, several of the demonstrated acoustic properties, including response at high frequencies (up to the measured $18 \mathrm{MHz}$ ), ability to produce harmonic signals (in Halo gas vesicles) and controlled acoustic collapsibility in situ, are especially advantageous for high-resolution contrast-enhanced imaging ${ }^{24}$. Future work is needed to determine how these properties are influenced by the specific size, shape, elasticity, gas diffusivity and collapse-resistance of these nanostructures. Some insights may be gained by refining existing microbubble theories, which predict peak responses at frequencies $>18 \mathrm{MHz}$ for contrast agents with submicrometre size and relatively high stiffness ${ }^{18,25-27}$. In addition, differences in the physical properties of gas vesicles obtained from distinct species, such as sharply tuned collapse pressures and harmonic scattering, may enable new detection schemes, as shown here with serial collapse imaging of gas vesicles from Ana and Halo.

Application-specific reporters can be generated by functionalizing the surface of gas vesicles through bioconjugation, as shown here with clustering-based analyte (streptavidin) detection by biotinylated Ana gas vesicles. The nanometre dimensions of these structures may enable the labelling of targets outside the bloodstream, such as tumours with increased vascular permeability ${ }^{28}$, to be explored in future studies. As with other nanoscale imaging agents, PEGylation or other surface treatments may be necessary to increase circulation time, reduce clearance by organs such as the liver, and decrease immunogenicity ${ }^{29}$. Future work is also needed to demonstrate the safety of gas vesicles for potential clinical and preclinical applications, including those that may require repeated administration. 

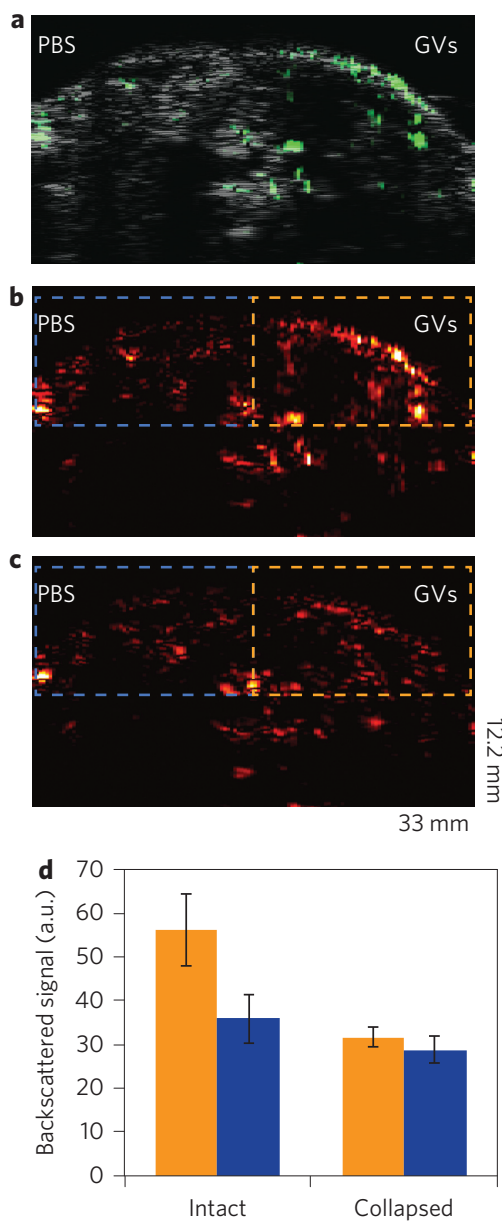
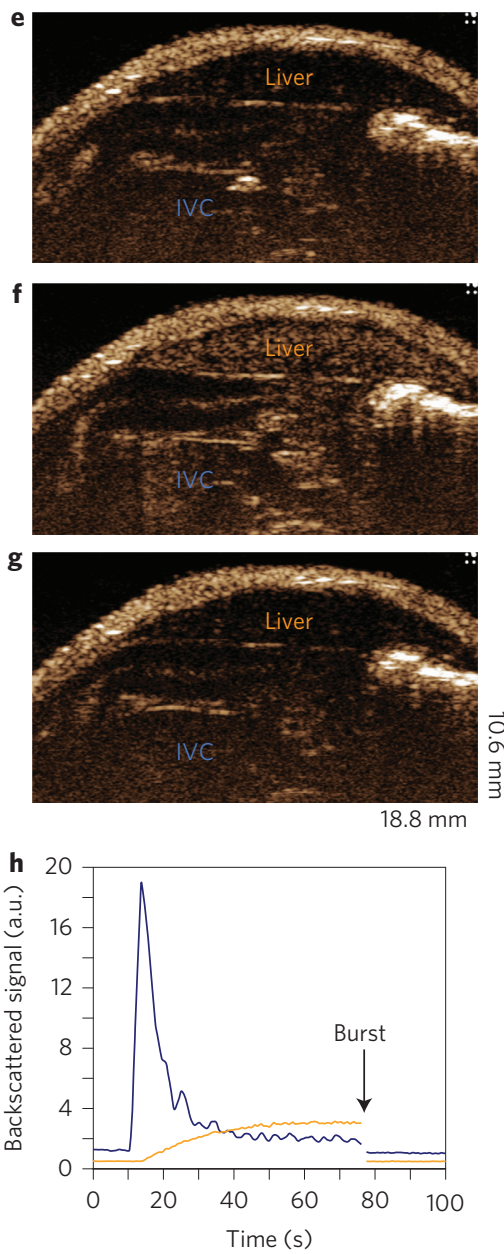
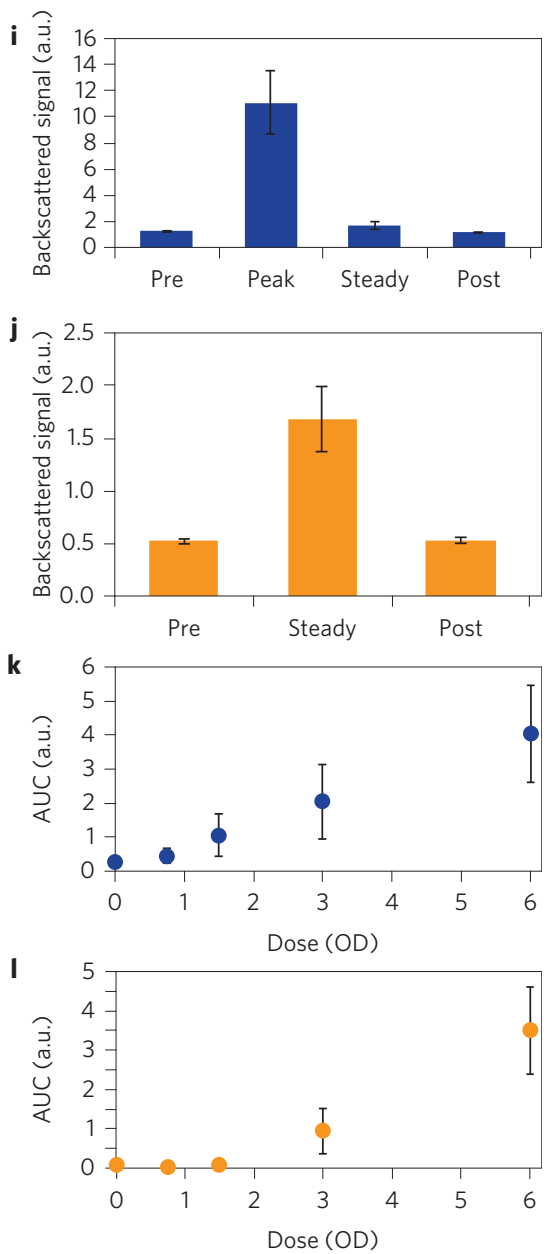

Figure 4 | Gas vesicles produce ultrasound contrast in vivo. a, Overlay of second-harmonic image ( $6 \mathrm{MHz}$ pulses) in green on a greyscale broadband anatomical image of mouse lower abdomen injected subcutaneously with $150 \mu$ l OD 6.0 Halo gas vesicles on the right side and $150 \mu$ l PBS on the left side. $\mathbf{b}, \mathbf{c}$, Second-harmonic ultrasound images before (b) and after (c) vesicle collapse with destructive insonation (650 kPa). Dashed outlines show regions of interest (ROI) used to quantify signals. d, Total backscattered second harmonic signal from ROls covering vesicle-injected (orange) and PBS-injected (blue) tissues, before and after collapse $(N=5)$. e- $\mathbf{g}$, Nonlinear contrast images acquired using a high-frequency ultrasound scanner system (operating at $18 \mathrm{MHz}$ and $2 \%$ power) of SCID nude mice infused intravenously with $50 \mu \mathrm{l}$ OD 6.0 Halo gas vesicles. The images show contrast at $4.5 \mathrm{~s}$ (e) and $64 \mathrm{~s}$ (f) after the start of infusion, or after the application of a burst pulse ( $\mathbf{g}$ ). Locations of the IVC and liver are indicated. $\mathbf{h}$, Time course of the smoothed average nonlinear signal in the IVC (blue) and liver (orange) during infusion. i, Mean average signal intensity in the IVC before (pre), during (peak) and after (steady) infusion, and after the burst pulse (post) $(N=5)$. j, Mean average signal intensity in the liver before (pre) and after (steady) infusion, and after the burst pulse (post) $(N=5)$. k,l, Dose-response relationship of $50 \mu$ l Halo gas vesicles infused at OD 0-6.0 determined from the AUC of average contrast in the IVC (k) and liver $(\mathbf{I})(N=5)$. Detailed image acquisition and analysis parameters are provided in Supplementary Table 1, and colour maps for ultrasound images in Supplementary Fig. 9. The size of each field of view is indicated in the lower right corner of the images. All error bars represent \pm s.e.m.

Intriguingly, the intracellular detectability of gas vesicles suggests that they could, in the future, be developed as reporter genes for bacterial or mammalian cell tracking, potentially enabling longer-term monitoring than recently demonstrated with microbubbles ${ }^{8}$. Indeed, gene clusters encoding gas vesicles have already been heterologously expressed in Escherichia coli ${ }^{30}$. Furthermore, genetic sequence control over the size, shape and collapse pressures of gas vesicles in their native hosts ${ }^{3}$ could assist with the optimization of gas vesicle properties for future use as either injectable or encoded reporters.

Finally, we note that the well-defined molecular structures, anisotropic shapes, hollow interiors, gas permeability, optical scattering, buoyancy, chemical reactivity, controlled collapse and genetic encoding of gas vesicles could conceivably make them useful in various other nanotechnology applications.

\section{Methods}

For additional details see Supplementary Information.
Gas vesicle preparation. Anabaena flos-aquae (Ana) was cultured in Gorham's media at room temperature under office fluorescent lighting. Halobacteria NRC-1 (Halo) was cultured with shaking at $37^{\circ} \mathrm{C}$ in ATCC medium 2185, under ambient light. Gas vesicles were isolated from Ana and Halo using hypertonic and hypotonic lysis, respectively, and purified by repeated centrifugally assisted flotation (Supplementary Fig. 8). Precollapsed gas vesicles were prepared through the application of hydrostatic pressure in a capped syringe.

The concentration of vesicles was estimated based on pressure-sensitive optical density at $500 \mathrm{~nm}\left(\mathrm{OD}_{500, \mathrm{PS}}\right)^{2}$. The relationship between $\mathrm{OD}_{500, \mathrm{PS}}$ and protein concentration was determined empirically using a total protein assay. Literaturebased estimates of molecular weight ${ }^{31-33}$ were used to calculate molar concentrations. Gas volume fractions were estimated using gas volumes of $8.4 \mu \mathrm{mg}^{-1}$ and $12.3 \mu \mathrm{l} \mathrm{mg}^{-1}$ for Ana and Halo gas vesicles, respectively ${ }^{32,33}$.

For experiments comparing intact and lysed cells, Ana cultures (cell $\mathrm{OD}_{600 \mathrm{~nm}} \approx 2$ ) were mixed 50:50 with either water or $50 \%$ sucrose for $60 \mathrm{~min}$ before imaging. Vesicle release was measured using $\mathrm{OD}_{500, \mathrm{PS}}$ and compared to dry cell pellet weight.

Biofunctionalization and aggregation. Ana gas vesicles were biotinylated using sulfo-NHS-LC-biotin and purified by three-times repeated flotation. For aggregation experiments, biotinylated vesicles were mixed with streptavidin at indicated molar ratios for $\sim 30$ min before imaging. 
In vitro ultrasound imaging. Imaging phantoms were prepared from $1 \%$ agarose in water. Two times concentrated gas vesicle samples were mixed 1:1 with melted 1\% agarose, and $100 \mu \mathrm{l}$ of the mixture was quickly loaded into phantom wells. The same procedure was used to load polystyrene microspheres ( $0.83 \%$ final wt/vol, $4.78 \mu \mathrm{m})$. Imaging was performed using a home-built imaging set-up. A 5, 10 or $20 \mathrm{MHz}$ single-element transducer $(6.3,6.3$ and $3.2 \mathrm{~mm}$ active areas, respectively; $25.4 \mathrm{~mm}$ focal distance) was mounted on a computer-controlled two-dimensional translating stage, immersed in water $\sim 20 \mathrm{~mm}$ above the sample. A programmable pulse generator and radiofrequency amplifier drove the transducers at specified frequencies with sinusoidal pulse trains of $\sim 1 \mu \mathrm{s}$. The preamplifier function of a pulse receiver with high-pass and low-pass filtering at $5 \mathrm{MHz}$ and $75 \mathrm{MHz}$, connected to an oscilloscope, was used to collect ultrasound signals and record them using MATLAB (Mathworks). In situ vesicle collapse was obtained by repeated pulsing at the indicated pressures at 8 or $8.6 \mathrm{MHz}$.

Image analysis. MATLAB was used to process raw time-domain data into twodimensional (B-mode) images. If multiple neighbouring lines perpendicular to the B-mode image were acquired, they were averaged. Regions of interest were defined manually in the axial dimension and automatically in the lateral dimension. Processing and scaling parameters are listed in Supplementary Table 1. Colour maps used in the images are shown in Supplementary Fig. 9. Power spectra represent squared absolute values of fast Fourier transforms of raw time-domain signals.

In vivo imaging. For imaging of subcutaneously injected gas vesicles (Fig. 4a-d) female CD-1 mice under isoflurane anaesthesia were depilated above the lower abdomen and injected subcutaneously with $150 \mu$ l Halo gas vesicles (OD 6) on one side and $150 \mu \mathrm{l}$ PBS on the other side of the abdomen. An ultrasonic transducer was coupled through a column of ultrasound gel above the injected region and scanned or used to apply destructive pulses as described above.

Intravenously injected gas vesicles were imaged in SCID nude mice using the VisualSonics Vevo 2100 high-frequency ultrasound scanner operating in nonlinear contrast mode, with the MS250 transducer set to $18 \mathrm{MHz}$ and $2 \%$ power. The mice were maintained under isoflurane anaesthesia on a heated imaging platform. Breathing and heart rate were monitored by built-in sensors. During infusion experiments, images were acquired continuously at a frame rate of 15 frames/s for $\sim 100 \mathrm{~s}$. A volume of $50 \mu \mathrm{l}$ Halo gas vesicles in PBS was infused $\sim 10 \mathrm{~s}$ after the start of the experiment at a flow rate of $0.6 \mathrm{ml} \mathrm{min}{ }^{-1}$. After $65 \mathrm{~s}$, a burst pulse was applied to collapse the vesicles. ROIs were analysed using Vevo Lab software. Smoothed infusion time-course curves were generated using locally weighed scatterplot smoothing. Area under the curve (AUC) values were obtained from raw data normalized to the pre-infusion baseline. Halo gas vesicles remaining in the liver following intravenous injection were imaged as described above, with the additional collection of images during $30 \mathrm{~s}$ windows at 15, 30, 45, 60 and/or $75 \mathrm{~min}$ after injection. In subcutaneous injection experiments, $100 \mu$ l OD 6.0 Halo gas vesicles were injected subcutaneously on the right side of the abdomen and $100 \mu \mathrm{l}$ PBS was injected contralaterally.

Veterinary evaluation. Animals were evaluated by a veterinarian on a 15-point scale comprising three points each for activity, weight, food intake, posture and hydration. The veterinarian was blinded to the injection group.

TEM. TEM images were obtained on a Philips/FEI Tecnai 12 microscope operating at $120 \mathrm{kV}$. Gas vesicle samples (OD 0.1) were deposited on a carbon-coated formvar grid and stained with $2 \%$ uranyl acetate.

Received 20 November 2012; accepted 28 January 2014; published online 16 March 2014

\section{References}

1. Smith-Bindman, R. et al. Use of diagnostic imaging studies and associated radiation exposure for patients enrolled in large integrated health care systems, 1996-2010. J. Am. Med. Assoc. 307, 2400-2409 (2012).

2. Walsby, A. E. Gas vesicles. Microbiol. Rev. 58, 94-144 (1994)

3. Pfeifer, F. Distribution, formation and regulation of gas vesicles. Nature Rev. Microbiol. 10, 705-715 (2012).

4. Gramiak, R., Shah, P. M. \& Kramer, D. H. Ultrasound cardiography-contrast studies in anatomy and function. Radiology 92, 939-948 (1969).

5. Ferrara, K., Pollard, R. \& Borden, M. Ultrasound microbubble contrast agents: fundamentals and application to gene and drug delivery. Annu. Rev. Biomed. Eng. 9, 415-447 (2007)

6. Kaufmann, B. A. \& Lindner, J. R. Molecular imaging with targeted contrast ultrasound. Curr. Opin. Biotechnol. 18, 11-16 (2007)

7. Cosgrove, D. \& Harvey, C. Clinical uses of microbubbles in diagnosis and treatment. Med. Biol. Eng. Comput. 47, 813-826 (2009).

8. Cui, W. et al. Neural progenitor cells labeling with microbubble contrast agent for ultrasound imaging in vivo. Biomaterials 34, 4926-4935 (2013).

9. Kircher, M. F., Gambhir, S. S. \& Grimm, J. Noninvasive cell-tracking methods. Nature Rev. Clin. Oncol. 8, 677-688 (2011).

10. Liu, J. et al. Nanoparticles as image enhancing agents for ultrasonography. Phys. Med. Biol. 51, 2179-2189 (2006).
11. Lanza, G. M. et al. A novel site-targeted ultrasonic contrast agent with broad biomedical application. Circulation 94, 3334-3340 (1996).

12. Martinez, H. P. et al. Hard shell gas-filled contrast enhancement particles for colour Doppler ultrasound imaging of tumors. Medchemcomm 1, 266-270 (2010).

13. Kripfgans, O. D., Fowlkes, J. B., Miller, D. L., Eldevik, O. P. \& Carson, P. L. Acoustic droplet vaporization for therapeutic and diagnostic applications. Ultrasound Med. Biol. 26, 1177-1189 (2000).

14. James, M. L. \& Gambhir, S. S. A molecular imaging primer: modalities, imaging agents, and applications. Physiol. Rev. 92, 897-965 (2012).

15. Klebahn, H. Gasvakuolen, ein Bestandteil der Zellen der wasserblutenbildenden Phycochromaceen. Flora (Jena) 80, 241-282 (1895).

16. Walsby, A. E. Pressure relationships of gas vacuoles. Proc. R. Soc. B 178, 301-326 (1971).

17. Burns, P. N. Harmonic imaging with ultrasound contrast agents. Clin. Radiol. 51, 50-55 (1996).

18. De Jong, N., Bouakaz, A. \& Frinking, P. Basic acoustic properties of microbubbles. Echocardiography 19, 229-240 (2002).

19. Hauff, P., Reinhardt, M. \& Foster, S. in Molecular Imaging I (eds Semmler, W. \& Schwaiger, M.) 223-245 (Springer, 2008).

20. Perez, J. M., Josephson, L., O’Loughlin, T., Hogemann, D. \& Weissleder, R. Magnetic relaxation switches capable of sensing molecular interactions. Nature Biotechnol. 20, 816-820 (2002).

21. Oliver, R. L. \& Walsby, A. E. Direct evidence for the role of light-mediated gas vesicle collapse in the buoyancy regulation of Anabaena flos-aquae (cyanobacteria). Limnol. Oceanogr. 29, 879-886 (1984).

22. Kashyap, S., Sundararajan, A. \& Ju, L. K. Flotation characteristics of cyanobacterium Anabaena flos-aquae for gas vesicle production. Biotechnol. Bioeng. 60, 636-641 (1998).

23. Geier, M. R., Trigg, M. E. \& Merril, C. R. Fate of bacteriophage lambda in non-immune germ-free mice. Nature 246, 221-222 (1973).

24. Foster, F. S., Pavlin, C. J., Harasiewicz, K. A., Christopher, D. A. \& Turnbull, D. H. Advances in ultrasound biomicroscopy. Ultrasound Med. Biol. 26, 1-27 (2000).

25. Frinking, P. J. \& de Jong, N. Acoustic modeling of shell-encapsulated gas bubbles. Ultrasound Med. Biol. 24, 523-533 (1998).

26. Hoff, L., Sontum, P. C. \& Hovem, J. M. Oscillations of polymeric microbubbles: effect of the encapsulating shell. J. Acoust. Soc. Am. 107, 2272-2280 (2000).

27. Medwin, H. Counting bubbles acoustically: a review. Ultrasonics 15, 7-13 (1977).

28. Yuan, F. et al. Vascular permeability in a human tumor xenograft: molecular size dependence and cutoff size. Cancer Res. 55, 3752-3756 (1995).

29. Jokerst, J. V., Lobovkina, T., Zare, R. N. \& Gambhir, S. S. Nanoparticle PEGylation for imaging and therapy. Nanomedicine 6, 715-728 (2011).

30. Li, N. \& Cannon, M. C. Gas vesicle genes identified in Bacillus megaterium and functional expression in Escherichia coli. J. Bacteriol. 180, 2450-2458 (1998).

31. Jost, M., Jones, D. D. \& Weathers, P. J. Counting of gas vacuoles by electron microscopy in lysates and purified fractions of Microcystis aeruginosa. Protoplasma 73, 329-335 (1971).

32. Walsby, A. E. \& Armstrong, R. E. Average thickness of the gas vesicle wall in Anabaena flos-aquae. J. Mol. Biol. 129, 279-285 (1979).

33. Yao, A. I. \& Facciotti, M. T. Regulatory multidimensionality of gas vesicle biogenesis in Halobacterium salinarum NRC-1. Archaea 2011, 716456 (2011).

\section{Acknowledgements}

The authors thank P. Lum for ultrasound equipment and advice, R. Zalpuri and K. McDonald for assistance with electron microscopy, K-K. Park and P. Khuri-Yakub for assistance with hydrophone measurements, E. Chérin for input on in vivo experiments and the manuscript, and A. Bar-Zion for assistance with data analysis. M.G.S. acknowledges funding from the Miller Research Institute and the Burroughs Wellcome Career Award at the Scientific Interface. Other funding was provided by California Institute for Regenerative Medicine grant RT2-02022 (D.V.S.), National Institutes of Health grant R01EB013689 (S.M.C), the Canadian Institutes of Health Research (F.S.F.) and the Terry Fox Foundation (F.S.F.).

\section{Author contributions}

M.G.S. conceived and directed the study, planned the experiments, prepared the specimens, collected, analysed and interpreted the data, and wrote the manuscript, with input from all other authors. P.W.G. designed and constructed the imaging instrument and accompanying signal processing software, and assisted with initial experiments. A.N. designed, constructed and optimized the imaging instrument and accompanying signal processing software. F.S.F. and M.Y. designed, performed and analysed the data from in vivo experiments. All authors provided input on the study and experimental design, data analysis, data interpretation and the manuscript.

\section{Additional information}

Supplementary information is available in the online version of the paper. Reprints and permissions information is available online at www.nature.com/reprints. Correspondence and requests for materials should be addressed to M.G.S.

\section{Competing financial interests}

F. Stuart Foster is a consultant to VisualSonics. 


\section{Biogenic gas nanostructures as ultrasonic molecular reporters}

Mikhail G. Shapiro*, Patrick W. Goodwill, Arkosnato Neogy, Melissa Yin, F. Stuart Foster, David V. Schaffer, Steven M. Conolly

\section{CONTENTS}

1. Supplementary Detailed Methods

2. Supplementary Table S1 - Imaging parameters

3. Supplementary Table $\mathrm{S} 2-$ Gas vesicle toxicity assessment

4. Supplementary Figure $S 1-$ Gas vesicle longevity

5. Supplementary Figure $\mathrm{S} 2$ - Attenuation effect from Halo gas vesicles

6. Supplementary Figure S3 - Harmonic response of Ana gas vesicles

7. Supplementary Figure S4 - Gas vesicle imaging in vivo

8. Supplementary Figure S5 - Effect of gas vesicle injection on heart rate and breathing

9. Supplementary Figure S6 - Gas vesicle clearance in the liver

10. Supplementary Figure S7 - Persistent gas vesicle signal after subcutaneous injection

11. Supplementary Figure S8 - Gas vesicle purification from Ana and Halo cells

12. Supplementary Figure $S 9$ - Colour maps used in ultrasound images

13. Supplementary Figure S10 - Representative regions of interest

14. Supplementary References 


\section{Supplementary Detailed Methods}

Gas vesicle preparation. Anabaena flos-aquae (Ana, strain 1403/13F, CCAP, Argyll, Scotland) was cultured in sterile Gorham's media at room temperature under office fluorescent lighting with an approximately $75 \%$ circadian duty cycle. Halobacteria NRC-1 (Halo, Carolina Biological Supply, Burlington, NC) was cultured at $37^{\circ} \mathrm{C}$ in high-salt medium (ATCC medium 2185), under ambient light, with rotation shaking at $225 \mathrm{rpm}$. GVs were isolated from Ana and Halo using hypertonic and hypotonic lysis, respectively (for 1 hour at room temperature), and purified by centrifugally-assisted flotation at $300 \mathrm{rcf}$ (Supplementary Fig. S8) Hypertonicity was achieved by adding sucrose to a final concentration of $25 \%$. Hypotonicity was implemented by diluting cells with a >8x volume of low-salt buffer $(10 \mathrm{mM}$ Tris- $\mathrm{HCl}, 2.5 \mathrm{mM} \mathrm{MgCl} 2,0.5 \mathrm{mM}$ $\mathrm{CaCl} 2, \mathrm{pH} 7.6)$. To achieve purity (> 6,400X enrichment vs. non-buoyant cellular components), the harvested GVs were twice resuspended in $>80 \mathrm{X}$ volumes of phosphate-buffered saline (PBS) and recentrifugated as above. This procedure is illustrated in Supplementary Fig. 5. GVs were diluted to experimental concentrations using PBS. To prepare pre-collapsed GVs, GV solutions were loaded into capped plastic syringes and the plunger depressed until the initially opaque solution (due to light scattering by intact GVs) became translucent (due to absence of light scattering by collapsed GVs).

The concentration of GVs was estimated based on their optical density (OD). Solutions of intact GVs appear milky white due to the scattering of light by their gas compartments ${ }^{1}$. GV collapse eliminates this light scattering, making it possible to subtract background contributions to the solution OD, yielding the "pressure-sensitive" OD at $500 \mathrm{~nm}\left(\mathrm{OD}_{500, \mathrm{PS}}\right)$. The relationship between $\mathrm{OD}_{500, \mathrm{PS}}$ and protein concentration (in $\mathrm{mg} / \mathrm{mL}$ ) was determined empirically using a total protein assay (micro-BCA kit, Pierce, Rockford, IL) performed on GVs purified as described above. Literature-based estimates of the molecular weight of the GVs (107 MDa for Ana, $686 \mathrm{MDa}$ for $\mathrm{Halo})^{2-4}$ were used to calculate the molar concentration. We obtained a value of $564.2 \pm 94.2 \mathrm{pM} / \mathrm{OD}_{500, \mathrm{PS}}(\mathrm{N}=5)$ and $16.1 \pm 3.2 \mathrm{pM} / \mathrm{OD}_{500, \mathrm{PS}}(\mathrm{N}=3)$ for Ana GVs and Halo GVs, respectively, which we rounded up to 600 and $20 \mathrm{pM} / \mathrm{OD}_{500, \mathrm{Ps}}$, respectively. The value for Ana GVs is slightly higher than $\sim 450 \mathrm{pM} / \mathrm{OD}_{500, \mathrm{PS}}$ which can be calculated from literature, the discrepancy possibly arising from incomplete GV purity, the presence of popped GVs in the OD measurement and/or differences in the protein assays used. Gas volume fractions were estimated using approximate gas volumes of $8.4 \mu \mathrm{L}$ per mg and $12.3 \mu \mathrm{L}$ per mg for Ana and Halo GVs, respectively, derived from literature ${ }^{3,4}$.

For experiments comparing intact and lysed cells, concentrated Ana cultures (cell $\mathrm{OD}_{600 \mathrm{~nm}} \sim 2$ ) were mixed 50:50 with either water or $50 \%$ sucrose for 60 minutes, then loaded into ultrasound imaging gels as described above. To measure GV release upon lysis, $\mathrm{OD}_{\mathrm{PS}, 500}$ was measured in solutions of lysed cells and compared to the dry pellet weight of paired cell samples (dry pellets were obtained by hydrostatically collapsing GVs inside the cells, pelleting the cells, washing with water, re-pelleting and drying overnight at $\left.80^{\circ} \mathrm{C}\right)$.

Bio-functionalization and aggregation. Ana GVs were biotinylated using EZ-Link Sulfo-NHS-LC-Biotin (Thermo Scientific, Rockford, IL) following supplier instructions, with a 10,000-fold molar excess of functionalizing reagent per GV, and purified by $3 \mathrm{X}$ repeated floatation (as in Supplementary Fig. S5c). For aggregation experiments, biotinylated GVs were mixed with streptavidin (G-Biosciences, St. Louis, MO) at indicated molar ratios and allowed to react for approximately 30 minutes before imaging.

In vitro ultrasound imaging. Imaging phantoms were prepared by casting $1 \%$ agarose gel (in water) around 96-well PCR reaction tubes, which were removed after solidification. 2X concentrated GV samples were mixed $1: 1$ with melted $1 \%$ agarose, and $100 \mathrm{uL}$ of the mixture was quickly loaded into phantom wells. The same procedure was used to load polystyrene microspheres $(0.83 \%$ final $\mathrm{w} / \mathrm{v}, 4.78 \mu \mathrm{m}$, Spherotech, Lake Forest, IL). After all samples have been loaded and solidified, additional $1 \%$ agarose was deposited to completely fill the wells and provide a uniform top surface. Imaging was performed using a home-built imaging setup. A $5 \mathrm{MHz}, 10 \mathrm{MHz}$ or $20 \mathrm{MHz}$ single element transducer $(6.3,6.3$ and $3.2 \mathrm{~mm}$ active areas, respectively; $25.4 \mathrm{~mm}$ focal distance; Olympus, Waltham, MA) was mounted on a computercontrolled 2D translating stage (VelMex, Bloomfield, NY). Transducer output was calibrated using a hydrophone (HGL-0200, Onda, Sunnyvale, CA). Phantoms were placed in a water container such that transducers could be immersed in the water at a distance of approximately $20 \mathrm{~mm}$ above the phantom. A programmable pulse generator (AFG3102, Tektronix, Beaverton, OR) and radio frequency amplifier (BT00500-AlphaS- CW, Tomco, Stepney, Australia) were used to drive transducers at specified frequencies with sinusoidal pulse trains of approximately $1 \mu \mathrm{s}$. The pre-amplifier function of a pulse-receiver (Pa- 
nametrics/Olympus 5601A/TT) with high-pass and low-pass filtering at $5 \mathrm{MHz}$ and $75 \mathrm{MHz}$, connected to an oscilloscope (Infiniivision DSOX2004A, Agilent Technologies, Santa Clara, CA; or LeCroy WaveJet 314 , Chestnut Ridge, NY) was used to collect ultrasound signals and record them using MATLAB (Mathworks, Natick, MA). In situ GV collapse was obtained by repeated pulsing at indicated pressures with a 10 $\mathrm{MHz}$ transducer operating at 8 or $8.6 \mathrm{MHz}$. Specific imaging parameters for each figure are listed in Supplementary Table S1.

Image analysis. Image analysis was performed in MATLAB. Raw time-domain signals acquired at each scan point were bandpass-filtered with symmetric Butterworth filters around the transmit or harmonic frequency, as indicated in Supplementary Table S1. After filtering, signals were brought back to baseband and time-gain compensation was applied using an empirically determined exponential coefficient. Line intensity profiles were obtained as the absolute value of a five point convolution of the in-phase and quadrature signal components. The resulting line intensity profiles were placed into arrays to generate twodimensional B-mode images. The time axis was converted to space using the speed of sound in aqueous media $(1540 \mathrm{~m} / \mathrm{s})$. If multiple neighboring lines perpendicular to the B-mode image were acquired, they were added together for averaging, as indicated in Supplementary Table S1. The resulting images were auto-scaled for presentation as indicated in Supplementary Table S1. Signals from regions of interest in each image were summated and used to generate quantitative plots. The regions of interest were defined manually in the axial dimension to include the contents of sample wells, and were defined automatically in the lateral dimension by detecting minima in axially integrated line intensity profiles and setting these minima as boundaries separating samples; the fidelity of automatically detected ROls was always confirmed visually (and found not to require adjustment). Additional processing parameters are listed in Supplementary Table 1. Colour maps used in the images are shown in Supplementary Fig. S9. Power spectra represent squared absolute values of fast Fourier transforms of raw time-domain signals from regions of interest corresponding to wells containing GVs or polystyrene (Fig 2a. is the average of 48 such spectra).

In vivo imaging. All animal protocols were approved by the Animal Care and Use Committee of the University of California at Berkeley and the Animal Care Committee of Sunnybrook Research Institute, as appropriate. For imaging of subcutaneously injected GVs, female CD-1 mice, maintained under isoflurane anesthesia throughout the experiment, were depilated above the lower abdomen and injected subcutaneously with 150uL Halo GVs (OD 6) on one side and 150uL PBS on the other side of the abdomen. Placed on their backs, the mice were fitted with a homemade container allowing a $\sim 3 \mathrm{~cm}$ column of ultrasound gel (Aquasonic, Parker Laboratories, Fairfield, NJ) to be placed above the injected region. An abdominal constriction was used to reduce breathing-related motion of the lower abdominal region. An ultrasonic transducer was coupled into the gel and scanned or used to apply destructive pulses as described above. To image animal anatomy (e.g., Fig. 4a), the pulse generation function of the pulsereceiver was used in place of the signal generator/amplifier. Additional scan parameters are listed in Supplementary Table S1.

Intravenously injected GVs were imaged in SCID nude mice using the VisualSonics Vevo 2100 high frequency ultrasound scanner operating in non-linear contrast mode, with the MS250 transducer set to 18 $\mathrm{MHz}$ and $2 \%$ power. The mice were maintained under isoflurane anesthesia and placed in supine position on a heated imaging platform. Breathing and heart rate were monitored by built-in sensors. The transducer was positioned above the abdomen such that the field of view would include the liver and the inferior vena cava. During infusion experiments, images were acquired continuously at a frame rate of 15frames/sec for approximately 100 seconds. $50 \mu \mathrm{L}$ of Halo GVs in PBS at the indicated concentrations were infused approximately 10 seconds after the start of the experiment at a flow rate of $0.6 \mathrm{~mL} / \mathrm{min}$. After 65 seconds, a burst pulse (100\% power) was applied for 1 second to collapse the GVs. To quantify contrast, ROls encompassing the inferior vena cava and liver were drawn manually in the Vevo Lab software (for example, see Supplementary Fig. S10), and the averaged non-linear contrast signal within each ROI for each frame was exported into MATLAB for further analysis. Smoothed infusion time course curves were generated from the raw signal using a locally weighed scatterplot smoothing algorithm ${ }^{5}$, which was further used to obtain pre-infusion, peak, steady state, and post-burst intensity values. To obtain AUC values, raw data was normalized to the average pre-infusion baseline, and positive signal values preceding the burst pulse were summated.

Halo GVs remaining in the liver following intravenous injection were imaged as described in the preceding paragraph, with the additional collection of images during 30 -second windows at $15,30,45,60$ 
and optionally 75 minutes after injection. The liver signal in each image was quantified using ROI analysis as described above.

To image the persistence of Halo GV contrast after subcutaneous injection, $100 \mu \mathrm{L}$ OD $6.0 \mathrm{Halo}$ GVs were injected subcutaneously on the right side of the abdomen of a SCID nude mouse and $100 \mu \mathrm{L}$ PBS was injected subcutaneously on the left side. The mouse was imaged using the Vevo 2100 system as described above. Images were collected immediately after injection and every 15 minutes thereafter for 120 minutes. A high-pressure burst pulse was applied to collapse any GVs still present after 120 minutes and imaged again.

Veterinary evaluation. The health of animals injected intravenously with OD 6.0 Halo GVs or PBS was evaluated by a veterinarian at Sunnybrook Research Institute on a 15-point scale comprising three points each for activity, weight, food intake, posture and hydration. The veterinarian was blinded to the injection group.

Transmission electron microscopy (TEM). TEM images were obtained on a Philips/FEl (Hillsboro, OR) Tecnai 12 microscope operating at 120kV. 10X diluted GV samples (from starting OD 1.0) were deposited on a carbon-coated formvar grid and negatively stained with $2 \%$ uranyl acetate in preparation for TEM imaging. 
Supplementary Table S1 - Image Acquisition and Analysis Parameters

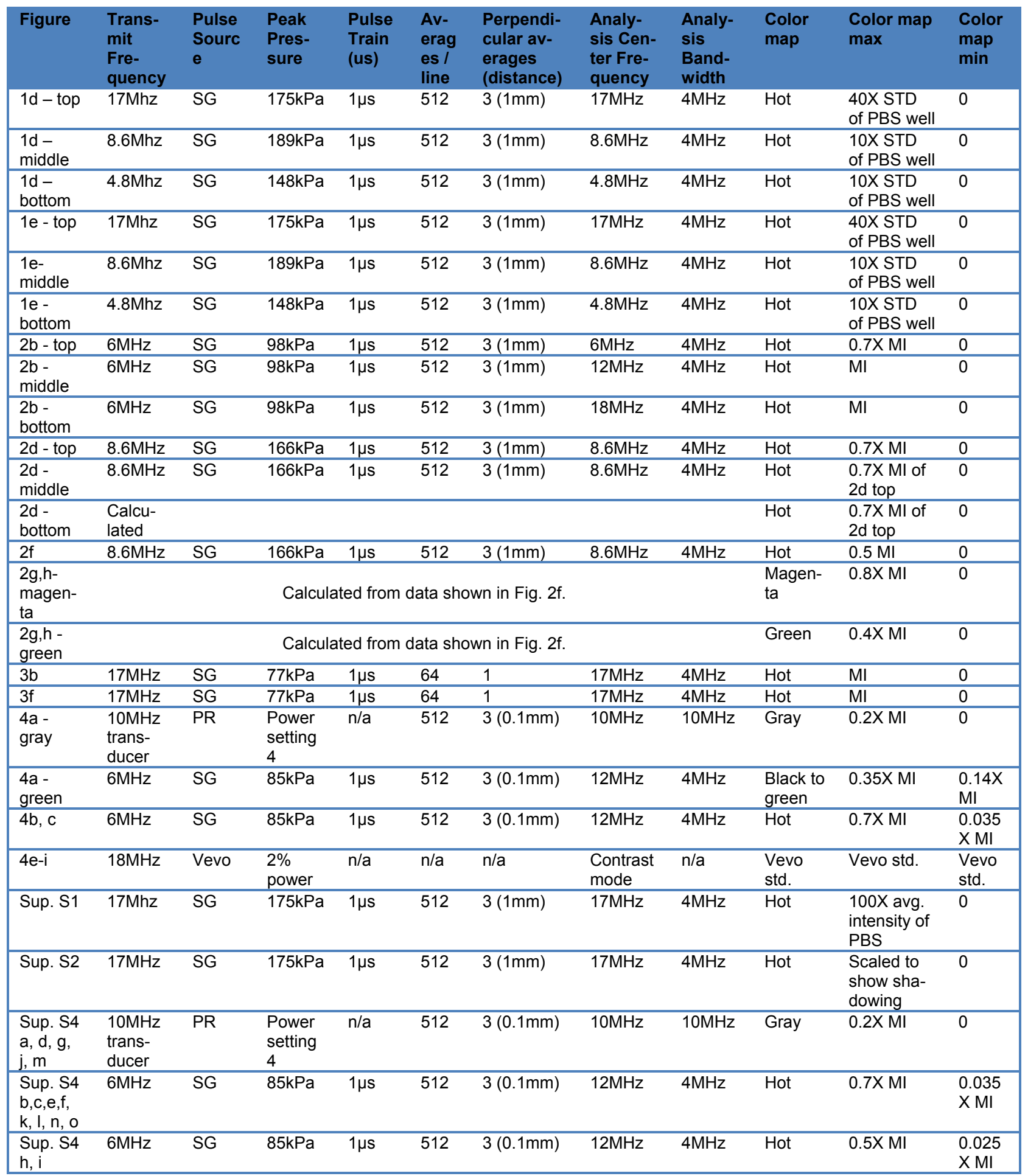

Abbreviations: SG - Signal generator, PR - Pulse receiver, Vevo - Vevo 2100 ultrasound scanner; STD - Standard deviation, MI - Maximum intensity 


\section{Supplementary Table S2 - Gas Vesicle Toxicity Assessment}

\begin{tabular}{|c|c|c|}
\hline & GVs & Saline \\
\hline Pre & 15 & 15 \\
\hline Post & 15 & 15 \\
\hline $\mathbf{2 4 h}$ & $14.7 \pm 0.3$ & 15 \\
\hline $\mathbf{4 8 h}$ & 15 & 15 \\
\hline
\end{tabular}

Supplementary Table S2 - Gas vesicle toxicity assessment. Mean health grades of mice injected intravenously with $50 \mu \mathrm{L}$ OD $6.0 \mathrm{Halo}$ GVs or PBS. Mice were scored on a 15-point scale comprising 3 points each for activity, weight, food intake, posture and hydration by a veterinarian blinded to injection group. Evaluations were performed before, immediately after and 24 and 48 hours after injection. ( $N=3$, \pm SEM). Only one mouse (at $24 \mathrm{~h}$ ) scored less than 15 points due to a minor drop in weight (resulting in a $14 / 15$ score), and recovered by $48 \mathrm{~h}$. 


\section{Supplementary Figure S1}

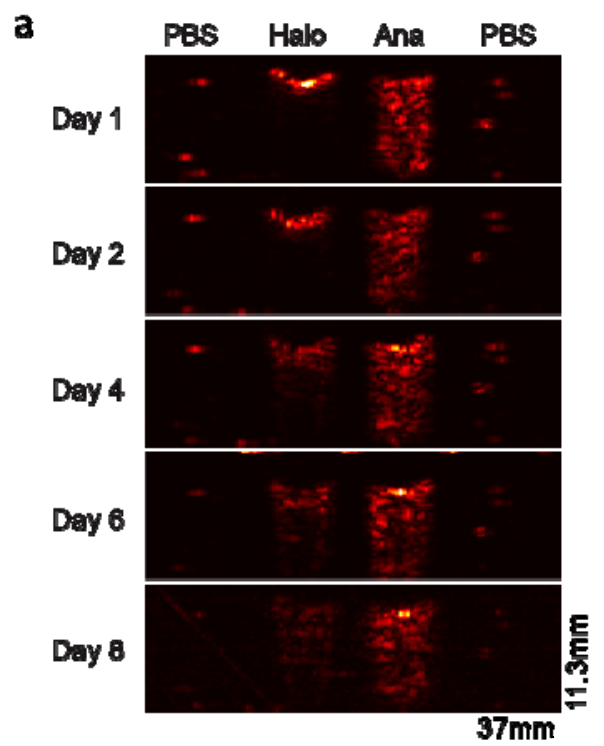

b

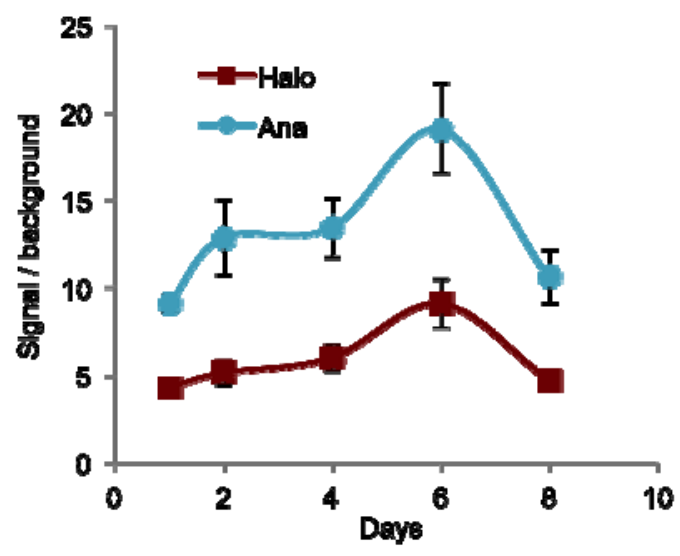

Supplementary Figure S1. Gas vesicle longevity. a, Ultrasound image (17 MHz pulses) of a phantom containing PBS buffer, OD 2.0 Halo GVs, OD 2.0 Ana GVs or another well of PBS buffer, acquired repeatedly over several days, as indicated. Note that the intensity profile of Ana GVs shows minimal change. Halo GV signal appears to show less of a shadowing effect, potentially indicative of a decrease in Halo GV concentration. b. Total backscattered signal from Halo and Ana GVs on each day of sampling, relative to PBS controls ( $\mathrm{N}=4 /$ sample). Detailed image acquisition and analysis parameters are provided in Supplementary Table S1; colour map in Supplementary Fig. S6. Error bars represent \pm SEM. The size of the field of view is indicated in the lower right corner of the bottom image. 


\section{Supplementary Figure S2}

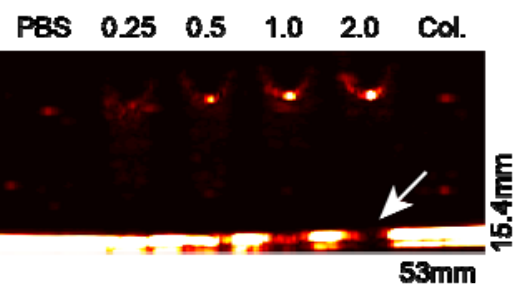

Supplementary Figure S2. Attenuation effect from Halo gas vesicles. Ultrasound image (17MHz) of phantom containing PBS buffer, Halo GVs at indicated optical densities or collapsed OD $2.0 \mathrm{Halo}$ GVs. The intense signal at the bottom of the image corresponds to the solid bottom of the imaging phantom. The arrow points to a location underneath Halo GVs where the signal from the solid bottom is attenuated. Smaller attenuation can be observed at lower concentrations of GVs. Detailed image acquisition and analysis parameters are provided in Supplementary Table S1; colour map in Supplementary Fig. S6. The size of the field of view is indicated in the lower right corner of the bottom image. 


\section{Supplementary Figure S3}

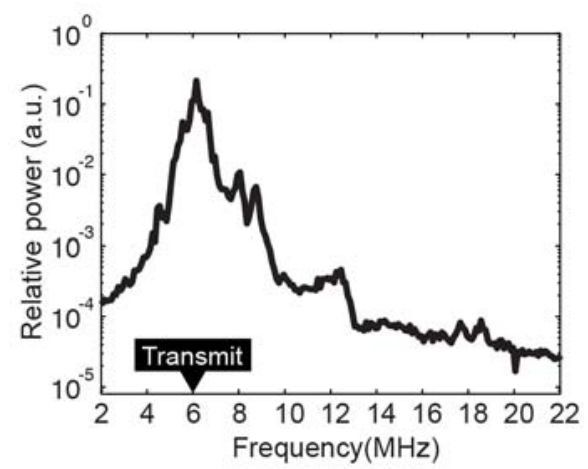

Supplementary Figure S3. Harmonic ultrasound response of Ana GVs. Power spectrum of signal backscattered from OD 2.0 Ana GVs in response to $6 \mathrm{MHz}$ ultrasound pulses (peak amplitude $98 \mathrm{kPa}$ ). Each point represents an average of 16 measurements. 


\section{Supplementary Figure S4}
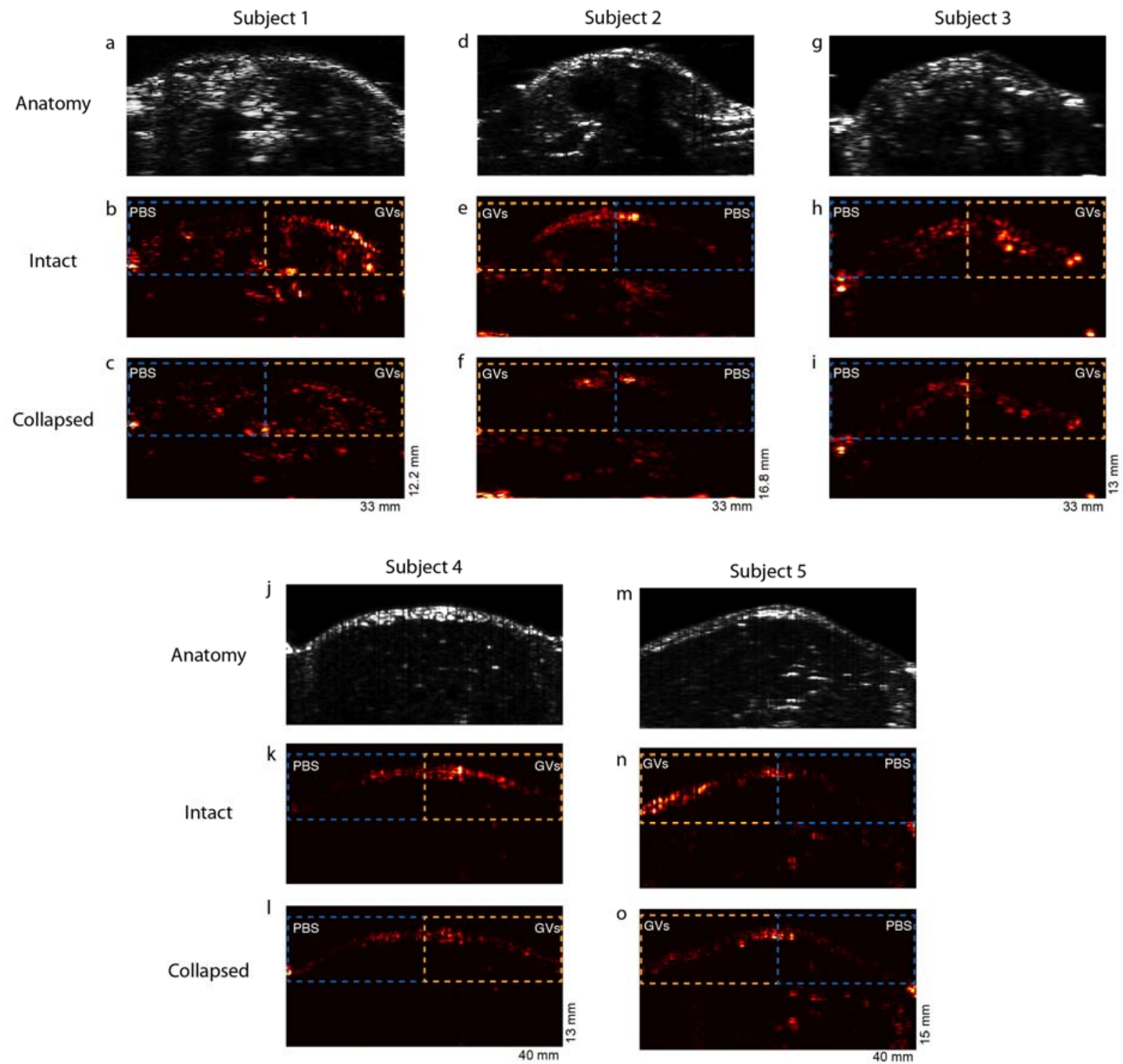

Supplementary Figure S4. Gas vesicle imaging in vivo. a, Three mice were depilated and injected subcutaneously with 150uL OD $6.0 \mathrm{Halo}$ GVs or 150uL PBS on opposite sides of the abdomen, as indicated. a, d, g, j m. Anatomical ultrasound images acquired with $10 \mathrm{MHz}$ transducer. $\mathbf{b}, \mathbf{e}, \mathbf{h}, \mathbf{k}, \mathbf{n} .12 \mathrm{MHz}$ harmonic ultrasound images $(6 \mathrm{MHz}$ pulsing) $\mathbf{c}, \mathbf{f}, \mathbf{i}, \mathbf{l}, \mathbf{0} .12 \mathrm{MHz}$ harmonic ultrasound images after GV collapse with destructive insonation. Dashed ovals indicate approximate location of injected material. Subject 1 is also shown in Figure 4. Detailed image acquisition and analysis parameters are provided in Supplementary Table S1; colour maps in Supplementary Fig. S6. The size of the field of view is indicated in the lower right corner of the bottom image. 


\section{Supplementary Figure S5}

a

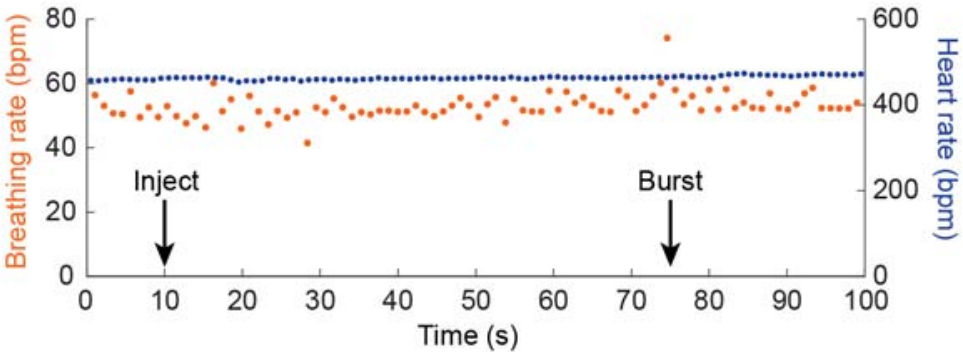

b
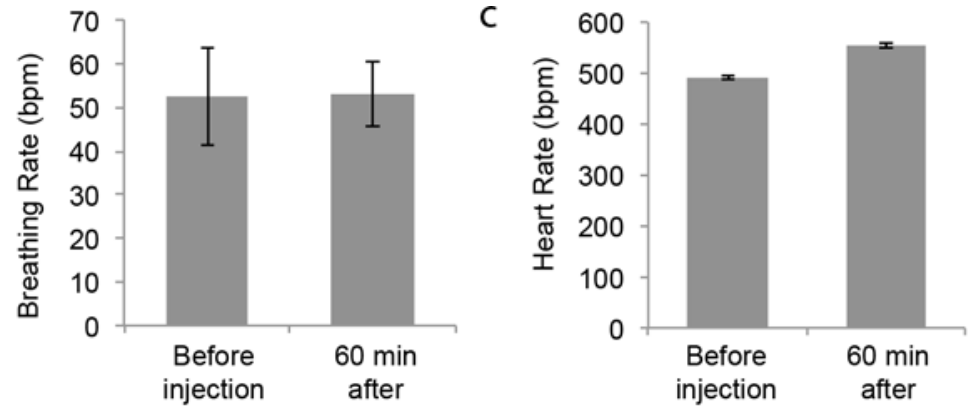

Supplementary Figure S5. Effect of gas vesicle injection on heart rate and breathing. a. Heart rate and breathing rate recorded during a Halo GV injection such as the one shown in Fig. 4, h. Arrows indicate the approximate timing of GV injection and the burst pulse. b-c. Mean heart rate (b) and breathing

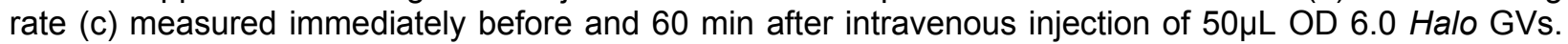
$\mathrm{N}=4$. Error bars $\pm \mathrm{SEM}$. 


\section{Supplementary Figure S6}

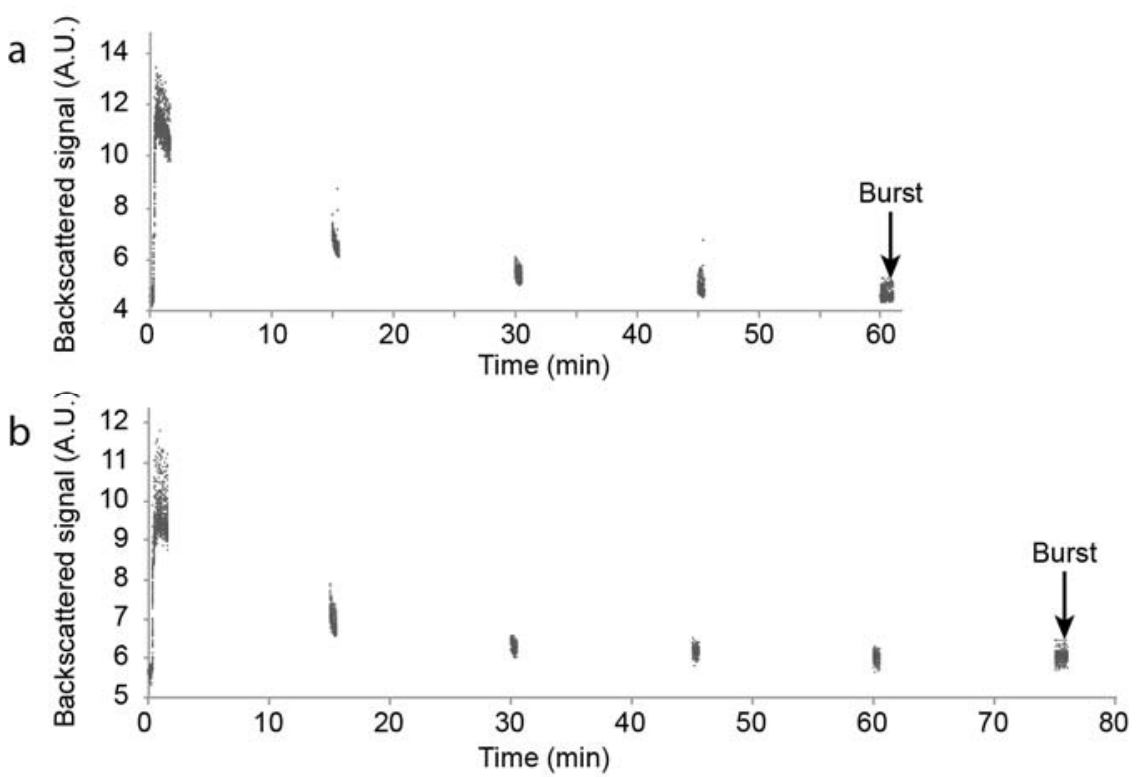

Supplementary Figure S6. Gas vesicle clearance in the liver. Backscattered non-linear signal in the livers of two mice (shown in a and b) injected intravenously with OD 6.0 Halo GVs. Injection, imaging parameters and ROI analysis were the same as for Fig 4, e-h, except that mice were kept anesthetized for 60-80 minutes after injection with imaging repeated every 15 minutes, as shown. At either 60 (a) or 75 (b) minutes after injection, a high-pressure burst pulse was applied to collapse any remaining GVs (as indicated by arrows). 


\section{Supplementary Figure S7}

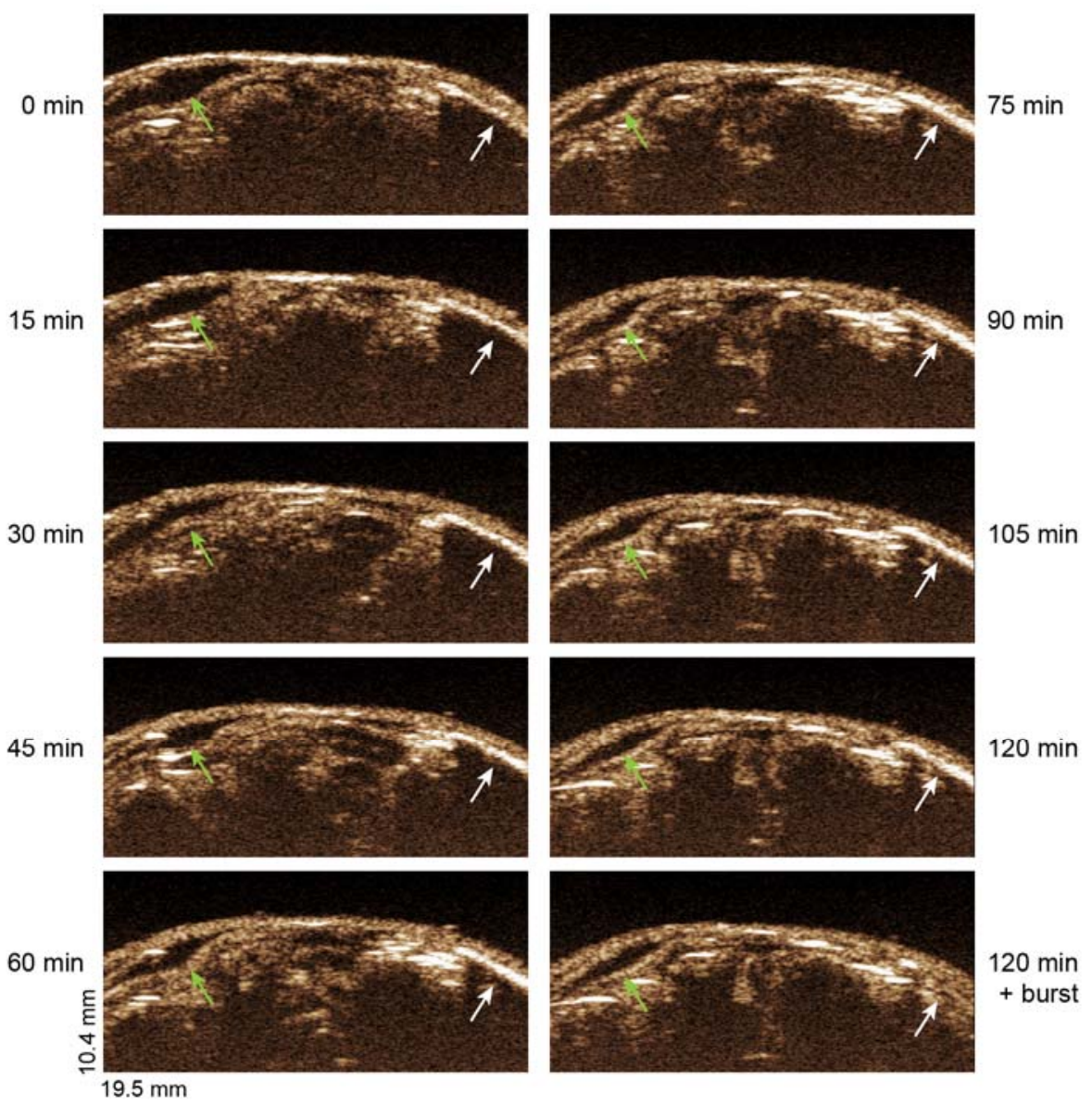

Supplementary Figure S7. Persistent gas vesicle signal after subcutaneous injection. Non-linear contrast images of SCID nude mice injected subcutaneously with $100 \mu \mathrm{L}$ OD $6.0 \mathrm{Halo}$ GVs (right side) or $100 \mu \mathrm{L}$ PBS buffer (left side), acquired using a high-frequency ultrasound scanner system operating at 18 $\mathrm{MHz}$ and $2 \%$ power. The images were taken immediately after injection and every 15 minutes thereafter as indicated next to each image. A high-pressure burst pulse was applied to collapse the GVs still present after 120 minutes, resulting in the bottom right image. The white arrow denotes contrast attributed to GVs (and its absence after the burst pulse). Note that the GVs show up as a bright anterior line of robust echogenicity with strong shadowing of the distal tissue below it due to attenuation (consistent with Supplementary Fig. S2). Brightening of the GV signal during the first hour of imaging is attributed to absorption of the PBS fluid in which the GVs are injected, increasing their local concentration; absorption of the fluid pocket is also observed on the control side. Both the highly echogenic signal and the shadowing are eliminated by the burst after $120 \mathrm{~min}$. The green arrow indicates the expected location of the injected PBS. 


\section{Supplementary Figure S8}

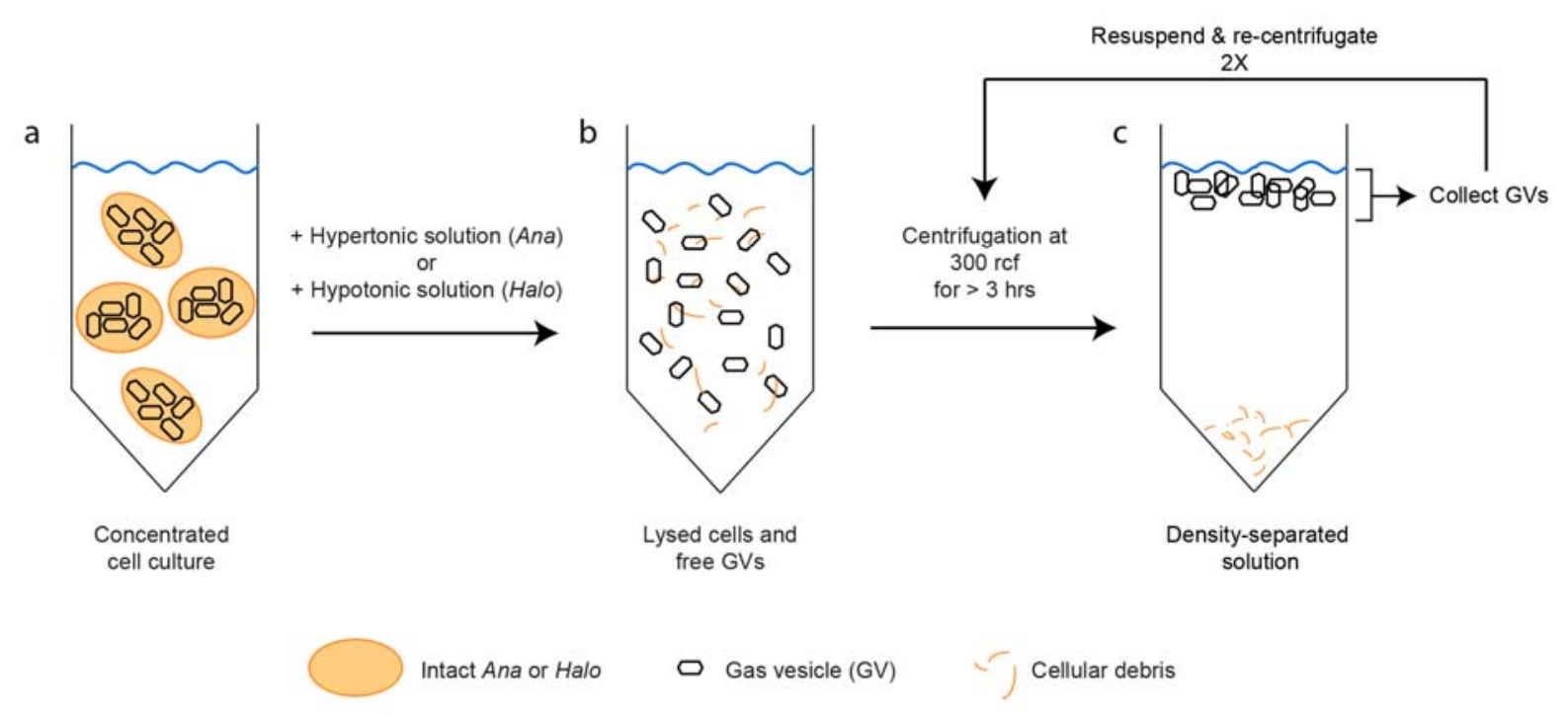

Supplementary Figure S8. Gas vesicle purification from Ana and Halo cells. GVs are purified through tonic lysis of cells followed by centrifugally-assisted floatation. a. Concentrated suspensions of Ana or Halo cells are disrupted through hypertonic lysis (addition of $25 \%$ sucrose) or hypotonic lysis (8fold dilution in low-osmolarity buffer), respectively, for 1 hour. $\mathbf{b}$. The resulting suspension is centrifugated for 3 hours or longer at $300 \mathrm{rcf}$ (this low speed is chosen to prevent GV disruption by pressure). c. GVs are collected from the surface of the centrifugated solution, re-suspended in $>80 \mathrm{X}$ PBS and recentrifugated. This step is repeated twice. 


\section{Supplementary Figure S9}

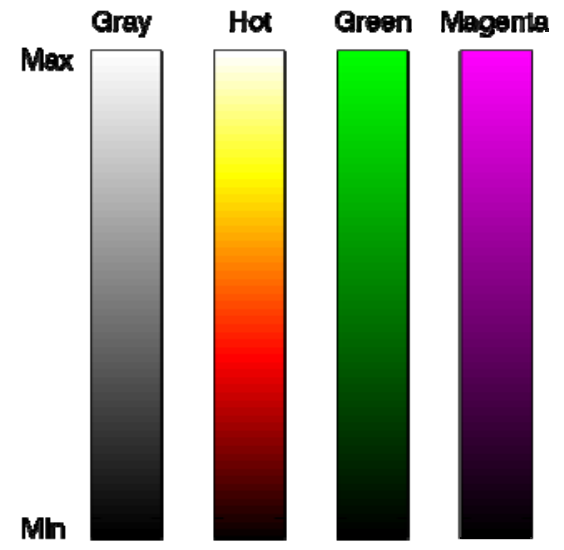

Supplementary Figure S9. Colour maps used in ultrasound images. Gray, Hot, Green and Magenta colour maps as used in Figs. 1-4 in the main text and Supplementary Figs. S1, S2 and S4. The scales are linear between Min and Max. The colour map used and the values of Min and Max are defined for each image in Supplementary Table S1. The colour maps Gray and Hot are standard in MATLAB. Green and Magenta were prepared by replacing the color white with either green or magenta in the Gray color map. 


\section{Supplementary Figure S10}
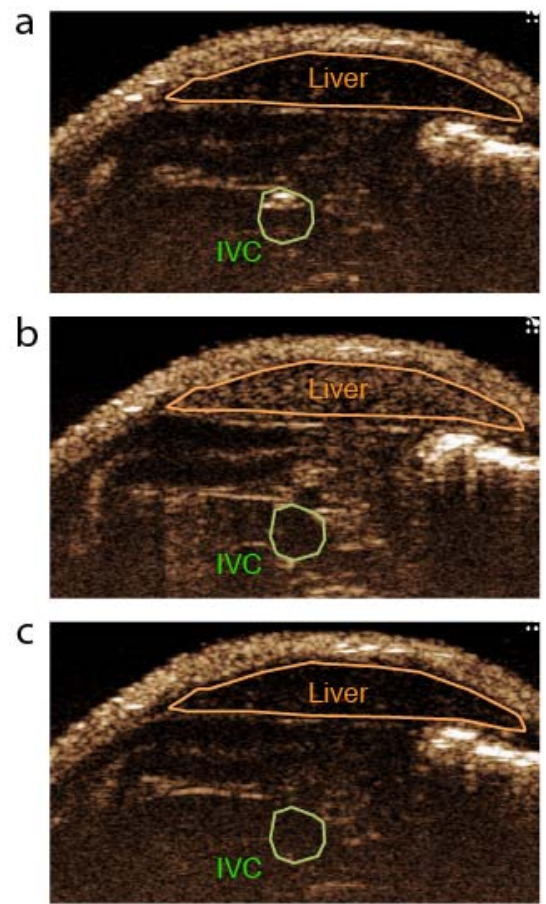

Supplementary Figure S10. Representative regions of interest used to generate Fig. 4, h-I. Nonlinear contrast images showing representative regions of interest (ROIs) for the liver and inferior vena cava (IVC), which were used to generate the data shown in Fig. 4, h-I.

\section{Supplementary References}

1. Walsby, A.E. Gas vesicles. Microbiol. Rev. 58, 94-144 (1994).

2. Jost, M., Jones, D.D. \& Weathers, P.J. Counting of gas vacuoles by electron microscopy in lysates and purified fractions of microcystis aeruginosa. Protoplasma, 329-335 (1971).

3. Walsby, A.E. \& Armstrong, R.E. Average thickness of the gas vesicle wall in Anabaena flos-aquae. J. Mol. Biol. 129, 279-285 (1979).

4. Yao, A.I. \& Facciotti, M.T. Regulatory multidimensionality of gas vesicle biogenesis in Halobacterium salinarum NRC-1. Archaea 2011, 716456 (2011).

5. Cleveland, W.S. Robust locally weighted regression and smoothing scatterplots. J. Am. Stat. Assoc. 74, 829-836 (1979). 